\title{
Importance of high IgG anti-Toxoplasma gondii titers and PCR detection of T. gondii DNA in peripheral blood samples for the diagnosis of AIDS-related cerebral toxoplasmosis: a case-control study
}

Authors

José E Vidal ${ }^{1}$

Adrián Vladimir

Hernández Diaz ${ }^{2}$

Augusto César Penalva de Oliveira $^{3}$

Rafi Felicio Dauar ${ }^{4}$

Fabio Antonio Colombo ${ }^{5}$

Vera Lucia Pereira-

Chioccola $^{5}$

${ }^{1}$ Department of Neurology,

Instituto de Infectologia

Emílio Ribas, São Paulo,

Brazil; AIDS Clinic, Schoo

of Medicine, Universidade

de São Paulo, São Paulo,

Brazil

${ }^{2}$ Department of

Quantitative Health

Sciences, Lerner Research

Institute, Cleveland Clinic,

Cleveland, USA

${ }^{3}$ Departament of Neurology,

Instituto de Infectologia

Emílio Ribas, São Paulo,

Brazil; Clinical Research

Unit in Retrovirology,

Universidade Estadual de

Campinas, São Paulo, Brazil

${ }^{4}$ Department of

Neurosurgery, Instituto de

Infectologia Emílio Ribas,

São Paulo, Brazil

${ }^{5}$ Department of

Parasitology, Instituto

Adolfo Lutz, São Paulo,

Brazil

Submitted on: $12 / 10 / 2010$ Approved on: 01/10/2011

Correspondence to: José E Vidal Av. Doutor Arnaldo, 165 01246900, Cerqueira César

São Paulo, Brazil josevibe@gmail.com

We declare no conflict of interest.

(ㅇ)2011 Elsevier Editora Ltda All rights reserved.

\begin{abstract}
Background: Cerebral toxoplasmosis (CT) continues to cause significant morbidity and mortality in human immunodeficiency virus (HIV)-infected patients in Brazil. In clinical practice, the initial diagnosis is usually presumptive and alternative diagnosis tools are necessary. Our objective was to evaluate whether the detection of high titers of IgG anti-Toxoplasma gondii and T. gondii DNA in blood samples are associated with the diagnosis of CT. Methods: In this case-control study we included 192 patients with HIV-1 infection: 64 patients with presumptive CT (cases) and 128 patients with other diseases (controls). Blood samples to perform indirect immunofluorescense reaction (IFI) to detect anti- $T$. gondii IgG antibodies and polymerase chain reaction (PCR) were collected before or within the first three days of anti-Toxoplasma therapy. Two multivariate logistic regression models were performed: one including the variable qualitative serology and another including quantitative serology. Results: In the first model, positive IgG anti-T. gondii (OR 4.7, 95\% CI 1.2-18.3; $\mathrm{p}=0.027$ ) and a positive T. gondii PCR result (OR 132, 95\% CI 35-505; $\mathrm{p}<0.001)$ were associated with the diagnosis. In the second model, IgG anti-T. gondii titres $\geq 1: 1024$ (OR 7.6, 95\% CI 2.3-25.1; $\mathrm{p}=0.001$ ) and a positive T. gondii PCR result (OR 147, 95\% CI 35-613; $\mathrm{p}<0.001$ ) were associated with the diagnosis. Conclusions: Quantitative serology and molecular diagnosis in peripheral blood samples were independently associated with the diagnosis of CT in HIV-infected patients. These diagnostic tools can contribute to a timely diagnosis of CT in settings where Toxoplasma infection is common in the general population.
\end{abstract}

Keywords: toxoplasmosis; cerebral toxoplasmosis; polymerase chain reaction; diagnosis; acquired immunodeficiency syndrome; Brazil.

\section{INTRODUCTION}

The introduction of highly active antiretroviral therapy (HAART) has resulted in a dramatic reduction of the incidence of acquired immunodeficiency syndrome (AIDS)-related cerebral toxoplasmosis (CT) in developed countries. ${ }^{1}$ Similarly, an important decrease in case reports have been observed in São Paulo in the HAART era. Nevertheless, CT continues to cause important mortality and morbidity in our settings. ${ }^{2}$ In clinical practice, presumptive diagnosis of CT is based on clinical and radiological features associated to successful response within 14 days of specific treatment., ${ }^{3,4}$ On the other hand, definitive diagnosis using biological tools (intraperitoneal inoculation to laboratory animals or inoculation to cell cultures in vitro) showed low sensitivity ${ }^{5}$ and brain biopsy has complications and elevated costs. ${ }^{6}$ Thus, alternative approaches for a timely diagnosis are necessary. In recent years, molecular diagnosis of CT in cerebrospinal fluid (CSF) and peripheral blood samples had significant improvement, ${ }^{5}$ but has not produced conclusive results. ${ }^{4}$ Here, we evaluated whether detection of high titers of IgG anti-Toxoplasma gondii and T. gondii DNA in blood samples could be used to diagnose CT.

\section{PATIENTS AND METHODS}

All patients were admitted to Instituto de Infectologia Emílio Ribas, a referral tertiary hospital in São Paulo, Brazil. This case-control study included 192 patients with HIV-1 infection documented by antibody enzyme-linked immunosorbent assay (ELISA) and Western Blot tests: 64 patients with presumptive cerebral toxoplasmosis (cases) and 128 patients with other 
diseases (controls). Cases were defined as follows: I) recent onset of a consistent focal neurological abnormality with intracranial disease or reduced level of consciousness; II) a lesion having a mass effect evidenced by brain imaging (on computed tomography or magnetic resonance) or a lesion whose radiographic appearance was enhanced by injection of contrast medium, and III) successful response to the specific treatment. The control group consisted of 128 patients with other diseases. Sixty-four presented with neurological diseases: 25 with cryptococcal meningoencephalitis, 7 with progressive multifocal leukoencephalopathy, 14 with central nervous system (CNS) tuberculosis, 12 with HIV-associated neurocognitive disorders, and six with syphilitic meningitis. The other 64 patients presented with non-neurological diseases: 18 with pulmonary tuberculosis, 12 with bacterial pneumonia, seven with oral candidiasis, five with diffuse lymphoma, and 22 with Pneumocystis jiroveci pneumonia. Blood samples to perform indirect immunofluorescense reaction (IFI) to detect anti-T. gondii IgG antibodies and polymerase chain reaction (PCR) were collected before or within the first three days of anti-Toxoplasma therapy. IgG $T$. gondii titers $\geq 1: 16$ and $\geq 1: 1024$ were considered positive and high, respectively. The samples were analyzed by conventional PCR targeting a 115-base-pair sequence in a specific repetitive region of the $B 1$ gene of T. gondii. PCR and IFI methodologies were reported elsewhere. ${ }^{7}$

For statistical analysis, the outcome variable in the present study was the diagnosis of CT. Univariate logistic regression analysis was performed to identify the independent variables that were associated with the diagnosis of CT. The list of potential variables included age, gender, prior CT, lymphocyte T-CD4+ cell count, use of CT prophylaxis at admission, positive IgG anti-T. gondii IFI, high titers of IgG anti-T. gondii IFI, and a positive PCR result for T. gondii DNA in blood samples. Only those variables with a p-value $\leq 0.2$ were included in the multivariate analysis. Variables with a p-value $\leq 0.05$ remained in the final multivariate logistic regression model. Correlations between qualitative serology (IFI $\geq 1: 16$ ) and quantitative serology ( $\geq 1: 1024)$, and between quantitative serology ( $\geq 1: 1024)$ and a positive T. gondii PCR were calculated with the nonparametric correlation Spearman's test. Statistical analysis was performed using SPSS software 10.0 (SPSS Inc. Product Registration, Chicago, USA, 1999). This study was approved by the institutional review board of Instituto de Infectologia Emílio Ribas.

\section{RESULTS}

The serological prevalence of T. gondii infection in the patients included in this study was $68 \%$ [58/64 (91\%) of cases and $72 / 128(56 \%)$ of controls $(\mathrm{p}<0.001)]$. When only patients with a positive result of IgG anti-T. gondii IFI we compared, cases $(52 / 58,90 \%)$ presented more frequently high IgG titers than controls $(39 / 72,54 \%)(\mathrm{p}<0.001)$.

According to the primary criteria in univariate analysis (Table 1), the following variables were associated with the

Table 1. Univariate analysis for the identification of independent variables associated with the diagnosis of cerebral toxoplasmosis

\begin{tabular}{|c|c|c|c|c|}
\hline Variable & Cases $(n=64)$ & Controls $(n=128)$ & OR (95\% CI) & p \\
\hline $\begin{array}{l}\text { Age (years) } \\
\quad \text { Mean } \pm \text { SD }\end{array}$ & $35.9 \pm 9.5$ & $35.8 \pm 7.7$ & $1.0(0.9-1.0)$ & 0.902 \\
\hline $\begin{array}{l}\text { Gender } \\
\text { Male } \\
\text { Female }\end{array}$ & $\begin{array}{l}37 \\
27\end{array}$ & $\begin{array}{l}78 \\
50\end{array}$ & $0.9(0.5-1.6)$ & 0.677 \\
\hline $\begin{array}{l}\text { Previous cerebral toxoplasmosis } \\
\text { Yes } \\
\text { No }\end{array}$ & $\begin{array}{l}11 \\
53\end{array}$ & $\begin{array}{c}16 \\
112\end{array}$ & $1.5(0.6-3.4)$ & 0.380 \\
\hline $\begin{array}{l}\text { CD4 T-cell count (cells/ } \mu \mathrm{L}) \\
\text { Mean } \pm \mathrm{SD}\end{array}$ & $71.4 \pm 72.9$ & $61.5 \pm 0.6$ & $1.0(0.9-1.0)$ & 0.284 \\
\hline $\begin{array}{l}\text { Use of prophylaxis at admission } \\
\text { Yes } \\
\text { No }\end{array}$ & $\begin{array}{c}3 \\
61 \\
\end{array}$ & $\begin{array}{c}23 \\
105\end{array}$ & $0.2(0.1-0.8)$ & 0.019 \\
\hline $\begin{array}{l}\text { IgG anti- } T \text {. gondii } \\
\quad \geq 1: 16 \\
\quad<1: 16\end{array}$ & $\begin{array}{c}58 \\
6 \\
\end{array}$ & $\begin{array}{l}72 \\
56\end{array}$ & $7.5(3.0-18.7)$ & $<0.001$ \\
\hline $\begin{array}{l}\text { IgG anti- } T \text {. gondii } \\
\quad \geq 1: 1024 \\
\quad<1: 1024\end{array}$ & $\begin{array}{l}52 \\
12\end{array}$ & $\begin{array}{l}41 \\
87\end{array}$ & $9.2(4.4-19.1)$ & $<0.001$ \\
\hline $\begin{array}{l}\text { T. gondii PCR in blood } \\
\text { Positive } \\
\text { Negative }\end{array}$ & $\begin{array}{l}51 \\
13\end{array}$ & $\begin{array}{c}3 \\
125\end{array}$ & $163(45-597)$ & $<0.001$ \\
\hline
\end{tabular}


diagnosis of CT: no use of CT prophylaxis at admission, $\operatorname{IgG}$ anti- $T$. gondii IFI $\geq 1: 16$ or IgG anti- $T$. gondii IFI $\geq 1: 1024$, and a positive T. gondii PCR result. Nonparametric correlations between qualitative and quantitative serology were high ( $\mathrm{p}<0.001)$. For this reason, were designed two multivariate logistic regression models: one including the variable qualitative serology and another including quantitative serology. In the first case, only positive T. gondii IFI [OR 4.7, 95\% CI 1.2-18.3; $\mathrm{p}=0.027]$ and a positive T. gondii PCR result [OR 132, 95\% CI 35-505; p < 0.001] remained in the final model. In the latter model, only IgG $T$. gondii IFI $\geq 1: 1024$ [OR 7.6, 95\% CI 2.3-25.1; $\mathrm{p}=0.001$ ] and a positive T. gondii PCR result [OR 147, 95\% CI 35-613; $\mathrm{p}<0.001]$ remained in the final model. In addition, nonparametric correlations between qualitative serology and a positive T. gondii PCR result, and quantitative serology and a positive T. gondii PCR result were high $(\mathrm{p}<0.001)$.

\section{DISCUSSION}

The main finding of our study, performed in a setting with high prevalence of Toxoplasma infection in the general population, was the significant association between high IgG anti-T. gondii titers and PCR detection of T. gondii DNA in peripheral blood samples and the diagnosis of CT.

Cerebral toxoplasmosis is the most frequent CNS opportunistic infection in HIV-infected patients in Brazil. ${ }^{8,9}$ In a previous report, we observed that $\mathrm{CT}$ accounts for $10 \%$ of the admissions at Instituto de Infectologia Emílio Ribas, usually occurring as AIDS-defining disease. ${ }^{2}$ In addition, similarly to other reports, ${ }^{10} \mathrm{CT}$ continue to appear in Brazil among patients with late HIV diagnosis, with poor compliance with HAART, or with treatment failure.

In the present study, we identified that non-compliance with CT prophylaxis at admission was predictive of CT in univariate analysis. The benefit of prophylaxis is well described in the literature; therefore, it is possible that this variable did not remain significant when included in the multivariate analysis due to the low proportion of controls using antibiotic prophylaxis at admission. Classically, positive IgG T. gondii serology has been considered in the diagnosis of CT. ${ }^{11}$ In a large study, Raffi et al. ${ }^{12}$ reported a trend towards higher median IgG anti-T. gondii titers in patients with CT $(\mathrm{p}=0.08)$. However, only qualitative $T$. gondii serology, but not the quantitative one, was associated to the diagnosis of CT. In accordance with studies that showed the predictive value of high IgG anti-T. gondii titers, ${ }^{13,14}$ our results showed an independent association between high titers of IgG anti-T. gondii and the diagnosis of CT. This association could reflect the direct effect of qualitative serology, as it was demonstrated with the high correlation between qualitative and quantitative serologies. Nevertheless, when we analyzed only the patients with a positive serology, cases had a frequency of elevated IgG titers higher than controls, suggesting the additional value of quantitative serology.
Available studies of PCR using peripheral blood in HIVrelated CT and usually performed with conventional PCR protocols and small number of subjects, reported sensitivities ranging from $13 \%$ to $88 \%$ with specificity $\geq 95 \%{ }^{5,15}$ Recently, we reported our experience with conventional PCR assay using peripheral blood samples in patients with AIDSrelated CT, showing a sensitivity of $80 \%$ and a specificity of $98 \% .^{7}$ These very divergent results can be due, at least in part, to several reasons: choice of target DNA and primers, PCR techniques, conditions and time of sample storage, and collection of blood samples before or in the first days after introduction of specific treatment. ${ }^{5,15,16}$ It was reported that genotypes of $T$. gondii strains isolated from patients with ocular toxoplasmosis in Brazil were highly divergent when compared to the previously described clonal lineages from North America and Europe, suggesting more frequent sexual recombinants resulting in mixed genotypes. ${ }^{17}$ Similarly, our group showed the high rate of genetic exchange in T. gondii strains isolated from HIV-infected patients with CT. ${ }^{18}$ Future studies should evaluate the relation between genetic diversity, parasitic burden, molecular diagnosis and clinical outcomes in AIDS patients with CT.

Introduction of real-time PCR and automated DNA extraction could avoid some limitations of conventional PCR and perform a more accurate and timely diagnosis. ${ }^{15}$ A recent report of our group found high sensitivity of realtime quantitative PCR, in CT diagnosis, using two different primer sets $(B 1 \mathrm{Tg}$, which amplified a sequence from the $B 1$ gene and RETg, which amplified a PCR product of the 529 base pairs sequence). ${ }^{19}$

Reinforcing the importance of molecular diagnosis using peripheral blood samples in immunedeficient patients, a study of patients with allogenic stem cell transplantation suggested that quantitative PCR assay may guide preemptive therapy and avoid death due to toxoplasmosis in about $80 \%$ of patients who develop infection..$^{20}$

International recommendations of diagnosis and management of expansive brain lesions in HIV-infected patients consider primary CNS lymphoma (PCNSL) as the second most frequent opportunistic disease in developed countries. ${ }^{4,21}$ However, PCNSL is uncommon in most developing countries, where focal forms of CNS tuberculosis (i.e. tuberculomas) are the main differential diagnosis of $\mathrm{CT}^{22,23}$ Furthermore, the clinical and radiological differentiation between these opportunistic infections poses important challenges in resource-limited settings, where both infections are highly prevalent in the general population and alternative and timely diagnosis approach are necessary. ${ }^{24}$

In accordance with a previous study carried out in a middle-income country, our findings demonstrate that PCR is a relatively simple and rapid procedure that can be performed in developing countries with reasonable laboratory infrastructure. ${ }^{25}$ The results of the present study highlight 
the importance of considering quantitative serology and molecular diagnosis in peripheral blood samples in the initial management of HIV-infected presenting with expansive brain lesions. In this line, a recent algorithm incorporated these tools. ${ }^{26}$

In conclusion, our results suggest that high IgG antiToxoplasma titers and a positive PCR result for of T. gondii DNA in blood samples were significantly associated with CT in AIDS patients. These diagnostic tools must be interpreted in association with clinical and radiological information, and can contribute to a timely diagnosis, especially in Brazil and in other low and middle-income countries with high prevalence rates of Toxoplasma infection in the general population.

\section{REFERENCES}

1. d'Arminio Monforte A, Cinque P, Mocroft A et al. Changing incidence of central nervous system diseases in the euro SIDA Cohort. Ann Neurol 2004; 55:320-8.

2. Vidal JE, Hernandez AV, de Oliveira AC et al. Cerebral toxoplasmosis in HIV-positive patients in Brazil: clinical features and predictors of treatment response in the HAART era. AIDS Patient Care STDS 2005; 19:626-34.

3. Pereira-Chioccola VL, Vidal JE, Su C. Toxoplasma gondii infection and cerebral toxoplasmosis in HIV-infected patients. Future Microbiol 2009; 4:1363-79.

4. Portegies P, Solod L, Cinque P et al. Guidelines for the diagnosis and management of neurological complications of HIV. Eur J Neurol 2004; 11:297-304.

5. Bastien P. Molecular diagnosis of toxoplasmosis. Trans R Soc Trop Med Hyg 2002; 96(Suppl.1):S205-15.

6. Skiest DJ. Focal neurological diseases in patients with acquired immunodeficiency syndrome. Clin Infect Dis 2002; 34:103-15.

7. Colombo FA, Vidal JE, Penalva de Oliveira AC et al. Diagnosis of cerebral toxoplasmosis in AIDS patients in Brazil: importance of molecular and immunological methods using peripheral blood samples. J Clin Microbiol 2005; 43:5044-7.

8. Oliveira JF, Greco DB, Oliveira GC et al. Neurological disease in HIV-infected patients in the era of highly active antiretroviral treatment: a Brazilian experience. Rev Soc Bras Med Trop 2006; 39:146-51.

9. Vidal JE, Penalva de Oliveira AC, Fink MC, Pannuti CS, Trujillo JR. AIDS-related progressive multifocal leukoencephalopathy: a retrospective study in a referral center in São Paulo, Brazil. Rev Inst Med Trop Sao Paulo 2008; 50:209-12.

10. Manzardo C, Del Mar, Ortega M et al. Central nervous system opportunistic infections in developed countries in the highly active antiretroviral therapy era. J Neurovirol 2005; 11(Suppl. 3):S72-82.
11. Collazos J. Opportunistic infections of the CNS in patients with AIDS. CNS Drugs 2003; 17:869-87.

12. Raffi F, Franck J, Pelloux $\mathrm{H}$ et al. Specific anti-toxoplasmic IgG antibody immunoblot profiles in patients with AIDS-associated Toxoplasma encephalitis. Diagn Microbiol Infect Dis. 1999;34:51-6.

13. Derouin F, Leport C, Pueyo S et al. Predictive value of Toxoplasma gondii antibody titres on the occurrence of toxoplasma encephalitis in HIV-infected patients. AIDS 1996; 10:1521-7.

14. Hellerbrand C, Goebel FD, Disko R. High predictive value of Toxoplasma gondii IgG antibody levels in HIV-infected patients for diagnosis of cerebral toxoplasmosis. Eur J Clin Microbiol Infect Dis 1996; 15:869-72.

15. Bretagne S. Molecular diagnosis in clinical parasitology and mycology: limits of the current polymerase chain reaction (PCR) assays and interest of the real-time PCR assays. Clin Microbiol Infect 2003; 9:505-11.

16. Weiss JB. DNA probes and PCR for diagnosis of parasitic infections. Clin Microbiol Rev 1995; 8:113-30.

17. Khan A, Jordan C, Muccioli C et al. Genetic divergence of T. gondii strains associated with ocular toxoplasmosis, Brazil. Emer Infect Dis 2006; 12:942-9.

18. Ferreira IM, Vidal JE, Costa-Silva TA et al. Toxoplasma gondii: Genotyping of strains from Brazilian AIDS patients with cerebral toxoplasmosis by multilocus PCR-RFLP markers. Exp Parasitol 2007; 118:221-7.

19. Mesquita RT, Ziegler AP, Hiramoto RM, Vidal JE, PereiraChioccola VL. Real-time quantitative PCR in cerebral toxoplasmosis diagnosis of Brazilian human immunodeficiency virus-infected patients. J Med Microbiol 2010; 59:641-7.

20. Martino R, Bretagne S, Einsele $\mathrm{H}$ et al. Early detection of Toxoplasma infection by molecular monitoring of Toxoplasma gondii in peripheral blood samples after allogeneic stem cell transplantation. Clin Infect Dis 2005; 40:67-78.

21. Evaluation and management of intracranial mass lesions in AIDS. Report of the Quality Standards Subcommittee of the American Academy of Neurology. Neurology 1998; 50:21-6.

22. Shankar SK, Mahadevan A, Satishchandra P et al. Neuropathology of HIV/AIDS with an overview of the Indian scene. Indian J Med Res 2005; 121:468-88.

23. Smego Jr, RA, Orlovic D, Wadula J. An algorithmic approach to intracranial mass lesions in HIV/AIDS. Int J STD AIDS 2006; 17:271-6.

24. Vidal JE, Dauar RF, Penalva de Oliveira AC. Utility of brain biopsy in patients with acquired immunodeficiency syndrome before and after introduction of highly active antiretroviral therapy. Neurosurgery 2008; 63:E1209.

25. Joseph P, Calderon MM, Gilman RH et al. Optimization and evaluation of a PCR assay for detecting toxoplasmic encephalitis in patients with AIDS. J Clin Microbiol 2002; 40:4499-503.

26. Vidal JE, Penalva de Oliveira AC. Alterações neurológicas Parte 1. Doenças neurológicas oportunistas em pacientes infectados pelo HIV. In: Infectologia Ambulatorial. Diagnóstico e Tratamento. Lauletta Lindoso JA, da Eira M, Casseb J, Carvalho de Mello e Silva AC. eds. São Paulo: Sarvier, 2008. 